\title{
Intelligent materials: a review of applications in 4D printing
}

\author{
Xin Li, Fianzhong Shang and Zhuo Wang \\ Department of Mechatronics and Automation, National University of Defense Technology, Changsha, China
}

\begin{abstract}
Purpose - The paper aims to promote the development of intelligent materials and the 4D printing technology by introducing recent advances and applications of additive layered manufacturing (ALM) technology of intelligent materials and the development of the 4D printing technology. Also, an arm-type ALM technology of shape memory polymer (SMP) with thermosetting polyurethane is briefly introduced.

Design/methodology/approach - This paper begins with an overview of the development and applications of intelligent materials around the world and the $4 \mathrm{D}$ printing technology. Then, the authors provide a brief outline of their research on arm-type ALM technology of SMP with thermosetting polyurethane.

Findings - The paper provides the recent developments and applications of intelligent materials and 4D printing technology. Then, it is suggested that intelligent materials mixed with different functional materials will be developed, and these types of materials will be more suitable for 4D printing.

Originality/value - This paper overviews the current developments and applications of intelligent materials and its use in 4D printing technology, and briefly states the authors' research on arm-type ALM technology of SMP with thermosetting polyurethane.
\end{abstract}

Keywords 4D printing, Additive layered manufacturing, Intelligent materials, Thermosetting polyurethane

Paper type General review

\section{Introduction}

Additive layered manufacturing (ALM) technology has been around for nearly three decades, but only in the past two years it has captured the imagination of millions of people. Industry, government and public awareness has reached a "tipping point", and now ALM's current impact and future potential are recognized. Now a new disruptive technology is on the horizon that may take ALM to an entirely new level of capability with profound implications for society, the economy and the global operating environment of government, business and the public. Programmable matter (PM), here described as $4 \mathrm{D}$ printing, has the economic, environmental, geopolitical and strategic implications of ALM while providing new and unprecedented capabilities in transforming digital information of the virtual world into physical objects of the material world. The fourth dimension in $4 \mathrm{D}$ printing refers to the ability for material objects to change form and function after they are produced, thereby intelligent materials will be the key issue.

Intelligent materials have been widely promoted as a key technology that will enhance all manners of novel products with unique capabilities. Indeed, in 2013, the World Economic Forum's Global Agenda Council on Emerging Technologies identified a class of intelligent materials, self-healing materials, as one of the top ten most promising technological trends that can help deliver sustainable growth in future decades and which are nearing large-scale deployment. Many intelligent materials and products are

The current issue and full text archive of this journal is available on Emerald Insight at: www.emeraldinsight.com/0144-5154.htm

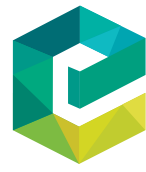

Assembly Automation

37/2 (2017) 170-185

Emerald Publishing Limited [ISSN 0144-5154]

[DOI 10.1108/AA-11-2015-093] available commercially, and the technologies continue to be the subject of worldwide academic research. However, there is a widely held view that existing intelligent materials could find far more widespread uses and the limited commercialized materials reflect in part the many misconceptions surrounding exactly what intelligent materials are and where they can use. One of the most influential ways of using intelligent materials is to incorporate them into ALM technology. This paper seeks to provide a broader review of this field and focuses on capabilities and applications (Aissa et al., 2012).

While ALM is becoming popular in increasingly more domains (Richards et al., 2013; Bogue, 2013; Bose et al., 2013; Wilhelm and Curbach, 2014; Frazier, 2014; Ivanova et al., 2013), the term of 4D printing has just been coined. This new dimension provides great potential to further widen the application areas of ALM technology. Although non-uniform material property itself may be defined as the

(C) Xin Li, Jianzhong Shang and Zhuo Wang. Published by Emerald Publishing Limited. This article is published under the Creative Commons Attribution (CC BY 4.0) licence. Anyone may reproduce, distribute, translate and create derivative works of this article (for both commercial \& non-commercial purposes), subject to full attribution to the original publication and authors. The full terms of this licence may be seen at http://creativecommons.org/licenses/by/4.0/legalcode

This paper forms part of a special section on Functionally Graded Rapid Prototyping, 4D Printing \& Self-Assemblies.

This work is supported by the NSFC (Natural Science Foundation of China): 51175505, National Ministries and Commissions Project: 51318010406, and the National University of Defense Technology Project (NUDTP): No. JC14-03-03 to Dr Zhuo Wang. The authors gratefully acknowledge the input of the anonymous reviewers.

Received 22 October 2015

Revised 16 May 2016

Accepted 1 June 2016 
additional dimension to ALM (Yamagishi et al., 2013), in this paper, this additional dimension only refers to the ability of a fabricated item to switch its geometric configuration (including surface morphology) from one to another in a fully controllable manner (Ge et al., 2014; Eujin, 2014a, 2014b; Tibbits, 2014; Tibbits and Cheung, 2012). A particular stimulus, such as heat (thermo-responsive), solution (chemo-responsive) and light (photo-responsive), may be applied to activate the switching process in either a reversible or a non-reversible process. As an example, well-known, traditional origami is a typical configuration/shape switching, but it is manual.

This paper reviews the development of intelligent materials systems around the world and the 4D printing technology, discuss what it is, investigate new applications that have been discovered and suggest its future impact. It can provide reference and help for future researchers. At the end of this paper, the authors provide a brief outline of independently developed arm-type ALM technology of shape memory polymers (SMPs) with thermosetting polyurethane matrix material. This technology is suitable for large-scale intelligent structure manufacturing because of its fast curing. Compared to other thermosetting polymers, the SMP material-based thermosetting polyurethane has the advantages of high heat-resistance temperature, big deformation recovery force and at low cost. It will have an important application prospect in space-deployable structure and morphing aircraft wing, etc.

\section{Intelligent materials in 4D printing}

Although widely discussed in the technical literature and also by the popular press, there is no universally accepted definition of exactly what "intelligent" materials are. Many maintain that they are materials which sense some stimulus from the external environment and create a useful response, but this would include conventional sensing materials such as piezoelectric or magnetostrictive compounds which several authors, indeed, categorize as "intelligent". However, a more useful view is to consider the response rather than the material itself.

Some authorities argue that there are no such things as intelligent materials per se but only materials that exhibit certain intrinsic characteristics which can be exploited in products, systems or structures that in turn exhibit "intelligent" behavior. Examples of this behavior include responses to external stimuli such as self-sensing, self-healing, self-actuating, self-diagnostic and shape-changing. Thus, intelligent materials can be seen as those which provide a means of achieving an active "intelligent" response in a product that would otherwise be lacking and have the potential to yield a multitude of enhanced capabilities and functionalities.

Examples of the capabilities of intelligent materials are as shown in Table I.

The fabrication methods and applications of intelligent materials are shown in Table II.

\subsection{Shape memory materials}

The shape memory effect (SME), as exhibited by metal alloys, such as nitinol, and many polymers, is defined as the ability, which has been quasi-plastically and severely pre-deformed, to recover initial shape under the right stimulus (Huang et al., 2010; Otsuka and Wayman, 1998). Typical stimuli to trigger the SME include heating/cooling (thermo-responsive) (in either direct or indirect manner), light (photo-responsive) (without involving much temperature fluctuation), chemicals (chemo-responsive) (e.g. water/moisture, ethanol, $\mathrm{pH}$ change), mechanical loading (mechano-responsive), etc., among others (Sun et al., 2012). Till date, the SME in a number of materials, which are known as shape memory materials (SMMs), have been applied in a wide range of applications, from biomedical devices (Huang et al., 2013) to $4 \mathrm{D}$ printing.

It should be pointed out that in some literature studies, the SME is confused with the shape change effect (SCE). The main difference between these two effects is that before applying the right stimulus, shape recovery in SMMs is limited, if there is any, while in the SCE, reversible shape change (either instantly or gradually) is dependent on whether the right stimulus is applied (Huang et al., 2012; Sun et al., 2012; Lendlein, 2010). For instance, while a piece of thermo-responsive shape memory alloy (SMA) requires heating to trigger the SME, elastic spring extends/contracts in response to force (mechano-responsive SCE). Because a same piece of material may have either the SME or the SCE depending on the working conditions, further confusion is highly likely. Traditional definition even worsens the situation. A typical example is thermo-responsive SMA, which, at low temperatures, has the SME, while, at high temperatures, is superelastic (according to current definition, this belongs to the SCE).

Fundamentally, the difference between the SME and SCE is due to the magnitude of the energy barrier between two shapes, one is permanent (A) and the other is temporary (B) as shown in Figure 1. If the energy barrier $(\mathrm{H})$ is high, additional energy (via applying the right stimulus) is required to overcome the barrier for shape recovery. This is the SME. On the other hand, if the energy barrier is low $\left(\mathrm{H}^{\prime}\right)$ or virtually none, the material is able to switch between these two shapes either gradually (e.g. viscoelastically) or instantly.

Table I The capabilities of intelligent materials

\begin{tabular}{lll}
\hline Function & Description & Status \\
\hline Shape memory & Material changes shape in response to some external stimulus & Established \\
Self-assembly & Allows automated folding for assembly & Established \\
Self-actuating & Automated actuation in response to an external stimulus & Research stage \\
Self-sensing & Allows automated detection and sometimes quantification of & Established, but is still the research focus
\end{tabular}


Table II Intelligent materials, their fabrication methods and field applications

\begin{tabular}{ll}
\hline $\begin{array}{l}\text { Intelligent materials } \\
\text { materials } \\
\text { Thermoresponsive }\end{array}$ & $\begin{array}{l}\text { Fabrication methods } \\
\text { Bonded together by the organic polymer point } \\
\text { by point }\end{array}$ \\
Athermally induced & $\begin{array}{l}\text { Photo-triggered }- \text { temporary shape is fixed by } \\
\text { covalently crosslinking cinnamic groups under } \\
\text { UV light of } \lambda>260 \text { nm and the shape } \\
\text { recovery is triggered by exposure to UV-light } \\
\text { irradiations of } \lambda<260 \text { nm and is water- } \\
\text { sensitive }- \text { When the polymer system is } \\
\text { exposed to water, deformation of the polymer } \\
\text { composite into a temporary shape takes } \\
\text { place. The shape fixing is done by subsequent } \\
\text { drying }\end{array}$
\end{tabular}

Self-assembly, selfactuating and selfsensing materials

\section{Electroactive \\ Polymer \\ IPMC}

Bucky Gel

DE
Applications

Aerospace - space-deployable structures (Fulcher et al., 2010 ), Biomedicine - stimuli-sensitive implant materials (Lendlein and Kelch, 2005), textiles - smart clothing (Mondal and Hu, 2006), and automobiles - fastening system

Biomedicine - endovascular thrombectomy device (Wu et al., 2010)

\author{
Biological applications, \\ Linear and rotary actuators, \\ Touch screens, \\ Underwater robots (Won et al., 2016), and biological imaging
}

Three-layered and five-layered IPMC structures solution is mixed as precursor materials, and mixed liquid of $\mathrm{Ag}$ fine particles and Nafion solution is used as electrode materials of IPMC

The electrode-base materials-electrode structure is solidified point by point accumulatively

Polyacrylic acid material is cured by UV light, then the surface of polyacrylic acid matrix material is coated with flexible electrode material
Figure 1 Shape memory effect and SCE

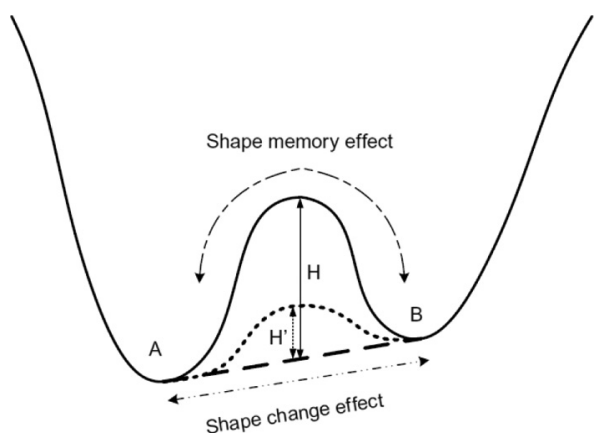

\subsubsection{Thermo-responsive SMMs}

Thermo-responsive SMMs are the most prominent class of SMMs used in 4D printing. There are two common thermos-responsive SMEs: one-way and two-way SMEs. One-way SME is that when a material is in its cold state, it can be bent or stretched and will hold those shapes until heated above the transition temperature. Upon heating, the shape changes to its original. When the material cools again, it will remain in the hot shape, until deformed again. The two-way SME is the effect that the material remembers two different shapes: one at low temperatures and one at high temperatures. A material that shows an SME during both heating and cooling is said to have a two-way shape memory. This can also be obtained without the application of an external force (intrinsic two-way effect). The reason the material behaves so differently in these situations lies in training. Training implies that a shape memory can "learn" to behave in a certain way. Figure 2 shows a scheme for showing the molecular mechanism of thermally induced SMM.

Carreño et al. proposed the ALM technology for SMA in 2007 (Carreño-Morelli et al., 2007). In their research, the 3D SMA structure was bound together by the organic polymer point by point. During the manufacturing process, a kind of solvent, which can react with the organic polymer, is sprayed into the mixture of NiTi metal powder and an organic adhesive, so that the NiTi metal powder can be bonded together. The material density of the SMA 
Figure 2 Molecular mechanism of SME

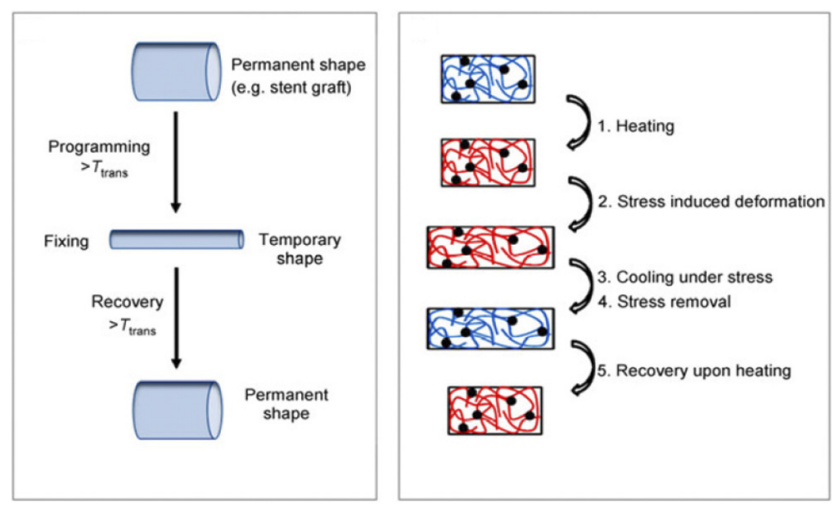

Notes: $\mathrm{T}_{\text {trans }}$ : shape memory transition temperature; black dots: net points; blue lines: molecular chains of low mobility below $\mathrm{T}_{\text {trans }} ;$ red lines: molecular chains of high mobility above $\mathrm{T}_{\text {trans }}$ Source: (Xie, 2011)

structure made by ALM technology has reached 95 per cent of the theoretical density and has an SME (Figure 3).

\subsubsection{Athermally induced SMMs}

Athermally induced SMEs such as photo-triggered actuation (Jiang et al., 2006; Lee et al., 2011) and water-triggered actuation are also ongoing research topics. Lendlein and Kelch (2005) developed the first light-induced SMMs by introducing photosensitive cinnamic acid into the polymeric network to serve as a molecular switch. With this approach, the temporary shape is fixed by covalently crosslinking cinnamic groups under UV light of $\lambda>260 \mathrm{~nm}$ and the shape recovery is triggered by exposure to UV light irradiations of $\lambda<260 \mathrm{~nm}$, in which covalent bonds formed during shape fixing are cleaved. Building upon this approach, Wu et al. (2010) developed a biodegradable light-sensitive SMM consisting of multi-block polyester urethanes that contain crystalline poly (L, L-lactide) hard and amorphous poly( $\varepsilon$-caprolactone) (PCL) soft segments and pendant cinnamamide moieties [Figure 4(a)]. Although nonheating-based light-activated SMM systems are attractive, the practical issues of low shape-fixity (20 - 55 per cent) and slow activation (60 - $120 \mathrm{~min}$ ), as well as the limited light penetration into the polymer bulk, must be resolved.

Figure 3 An SMA structure made by ALM technology and its SME

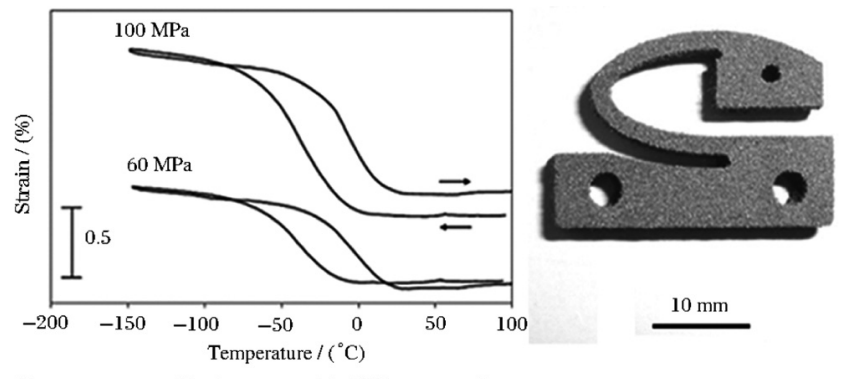

Shape-memory effects measured in NiTi processed NiTi part processed by 3D-printing by solvent on granule printing

Source: Carreño-Morelli et al. (2007)
Recently, another athermally induced SME has been reported for a water-sensitive SMM system comprising cellulose nanowhiskers (CNWs) and thermoplastic polyurethane (Zhu et al., 2012). In this system, the SME phenomenon is attributed to the reversible formation and disruption of the CNW percolation network upon exposure to water. When the polymer system is exposed to water, the presence of water molecules weakens the hydrogen bonds between the nanowhiskers; this effect allows easy deformation of the polymer composite into a temporary shape. The shape fixing is done by subsequent drying, in which the re-established hydrogen-boned 3D network of CNW can prohibit chain mobility. In the recovery stage, water is used to trigger spontaneous shape recovery due to decoupling of the CNW network. Figure 4(b) shows the water-sensitive shape memory mechanism for the $\mathrm{CNW} /$ polyurethanes system. Similar to the solvent-induced SME, the rate of shape recovery in this athermal water-sensitive system may vary according to the geometric shape of the device.

\subsection{Self-assembly, self-actuating and self-sensing materials}

The concept of "self-assembly" has been used interchangeably with 4D printing (Eujin, 2014b). However, for this paper, the author defines $4 \mathrm{D}$ printing as the process of ALM technology using appropriate intelligent materials, laying down successive layers of stimuli-responsive materials or multi-materials with different properties. After being built, the object reacts to stimuli from the natural environment or through human intervention, resulting in a physical or chemical change of state through time. This means that the end result of $4 \mathrm{D}$ printing is not limited to self-assemblies but also other states of change. Currently, self-assembly is almost used in microcosmic aspect, especially in biomedical field. Zhang (2013) has focused on fabricating several self-assembling peptides and proteins for a variety of studies and biomaterials (Figure 5). The examples include ionic self-complementary peptides (Zhang, 2011, 2013; Zhang et al., 2012), which form $\beta$-sheet structures in aqueous solution with two distinct surfaces - one hydrophilic and the other hydrophobic (rather like the pegs and holes in Lego bricks). The hydrophobic residues shield themselves from water and self-assembly in water in a manner similar to that seen in the case of protein folding in vivo.

In Figure 5(a), the ionic self-complementary peptide has 16 amino acids, nearly $5 \mathrm{~nm}$ in size, with an alternating polar and nonpolar pattern. The peptides form stable $\beta$-strand and $\beta$-sheet structures; thus, the side chains partition into two sides, one polar and the other nonpolar (Zhang, 2013; Holmes et al., 2010). They undergo self-assembly to form nanofibers with the nonpolar residues inside. Figure $5(\mathrm{~b})$ is a type of surfactant-like peptide, nearly $2 \mathrm{~nm}$ in size, that has a distinct head-charged group, either positively or negatively charged, and a nonpolar tail consisting of six hydrophobic amino acids. The peptides can self-assemble into nanotubes and nanovesicles with a diameter of 30-50 $\mathrm{nm}$. These nanotubes go on to form an interconnected network similar to what has been observed in carbon nanotubes. Figure 5(c) shows the surface nanocoating peptide. This type of peptide has three distinct segments: a functional segment, which interacts with other proteins and cells; a linker segment that not only can be 
Figure 4 Athermal SME

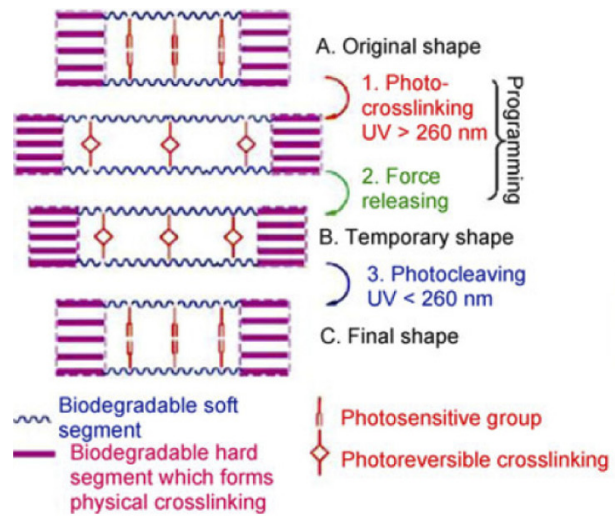

(a)

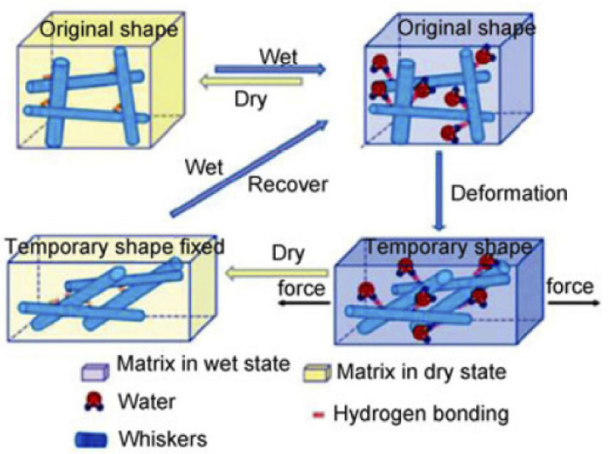

(b)

Notes: (a) Photo-responsive SME; (b) water-sensitive SME

Sources: Wu et al. (2010) and Zhu et al. (2012)

Figure 5 Fabrication of various peptide materials

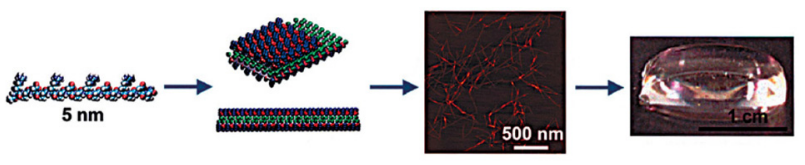

(a)

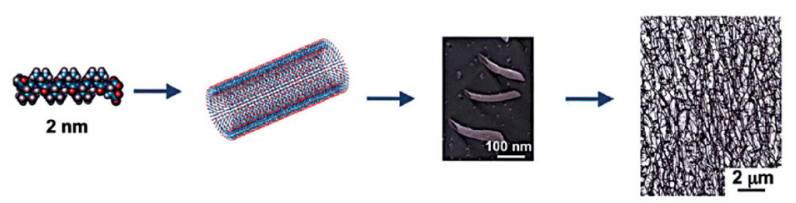

(b)

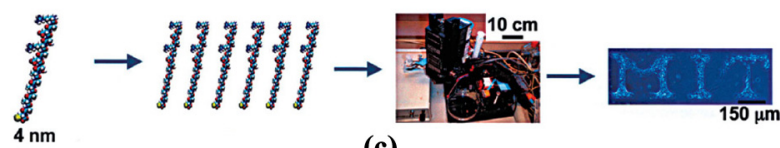

(c)

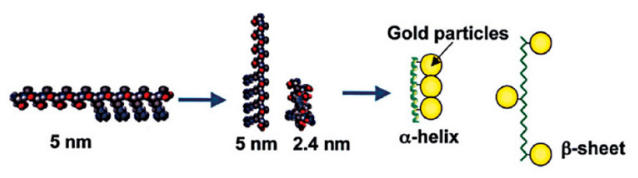

(d)

flexible or stiff but also can set the distance from the surface; and an anchor for covalent attachment to the surface. These peptides can be used as ink for an inkjet printer to print directly on a surface, instantly creating any arbitrary pattern. Figure $5(d)$ is the molecular switch peptide, a type of peptide with strong dipoles that can undergo drastic conformation changes, between $\alpha$-helix and $\beta$-strand or $\beta$-sheet, under external stimuli.

Self-actuation materials are closely allied to SMMs and involve the use of materials that create a significant strain or displacement in response to an external stimulus. The technology remains at the research stage, and composites containing piezoelectric elements, magnetostrictive elements, SMAs and electrorheological fluids are examples of experimental materials that exhibit this property. Ultimately, it is hoped that components and structures will be developed that can generate large displacements when subjected to electrical, thermal or mechanical stimuli. Although not self-actuating, SMAs have been used in linear and rotary actuators (see Applications, below).

Self-sensing underpins several intelligent functionalities and involves imparting sensing capabilities to materials which do not inherently possess this property, such as concrete, plastics, textiles, paper, metals and composites, and can be used to implement self-diagnosis, self-healing or other types of intelligent behavior. It can be achieved through the use of arrays of embedded sensing elements, the incorporation of sensing materials into other materials or so-called "sensing skins" which can be used to coat a structure or component. Self-sensing technology can potentially exploit a wide range of sensory phenomena, which create an electrical or other (e.g. colorimetric/visual) change. Fiber Bragg grating (FBG) sensors that are a class of distributed fiber-optic sensors have attracted much interest as the fibers can be incorporated relatively readily into composites, elastomers and polymers. Sensing skins are attracting much interest from the research community and in addition to using FBG sensors, perhaps the most widely studied approach, prototypes have recently been developed at the Georgia Institute of Technology which use the so-called "piezophototronic" effect, whereby touching the skin generates light through the use of pixels comprising arrays of miniaturized $\mathrm{n}-\mathrm{ZnO}$ nanowire/p-GaN LEDs. The arrays can detect pressure changes as small as $10 \mathrm{kPa}$, which is similar to a gentle tap with a finger and could find uses in touchscreens, robotics and biological imaging.

\subsection{Electroactive polymer}

Electroactive polymer (EAP) is a sort of new flexible functional materials that can produce significant changes in either size or shape under electrical field excitation. It is an important branch of intelligent materials (Chen et al., 2013). Ionic polymer-metal composites (IPMC), Bucky Gel and dielectric elastomers (DEs) are all EAPs. Manufacturing 3D 
complex structure of an electroactive polymer is an important research topic in the field.

\subsubsection{IPMC intelligent materials}

IPMC is a sort of composite material, which is generated in the process of electrode preparing. It sticks on both surfaces of ion exchange membrane. Under the effect of external voltage, the ions and water molecules inside the material gather to the electrode side, causing an imbalance in the distribution of mass and charge. So, bending deformation is generated at the macro level (Chen et al., 2013). Most of the IPMC produced by traditional methods are sheet slices (Bar and Yoseph, 1998). And for this reason, it is difficult to produce complex shapes of IPMC intelligent materials.

It was in 2006 that Evan Malone and Hod Lipson presented a method for preparing three- and five-layered structures IPMC by ALM (Malone and Hod, 2006). They mixed the Nafion solution and alcohol-water solution as precursor materials of the IPMC matrix, and used the mixed liquid of $\mathrm{Ag}$ fine particles and Nafion solution as electrode materials of IPMC. First, a silicone cube container is made by ALM, and then the electrode-Nafion matrix-electrode structure is solidified point by point accumulatively. The silicone cube container can provide the supports for IPMC manufacturing. It can prevent the extruded liquid from flowing before solidification. To reduce the solution evaporation and extend the life of IPMC intelligent materials, Malone and Hod (2006) made an improvement on the basis of a three-layered IPMC. They added a low conductive and impermeable electrode protection layer, which is made of Hydrin C thermoplastic (Zeon Chemicals L. P.), outside the solidified electrode by ALM. Five-layered IPMC by ALM can seal the solution in itself, which effectively extends the life of IPMC. Figure 6 shows the structure diagram of IPMC and the real IPMC made by ALM.

Although it has a large difference between sheet IPMC made by ALM and that by the traditional method, this kind of new ALM technology with intelligent IPMC material has laid a foundation to make a complex IPMC structure, which made the direct IPMC ALM technology come true.

\subsubsection{Bucky Gel intelligent material actuator/sensor}

Bucky Gel is one of the latest developed ionic EAP intelligent material. The composition, driving and sensing principle of Bucky Gel, is similar to that of IPMC. Bucky Gel contains three layers: the middle base material is an electrolyte layer that consists of a polymer and an ionic liquid and both sides of the base material are electrode materials that consist of carbon nanotubes, polymers and ionic liquid (Karl et al., 2014). When the voltage is applied on both sides of electrodes, cation and anion of the ionic liquid move to them, which cause the banding of Bucky Gel.

The traditional method for preparing Bucky Gel is solution casting method. The base layer and the electrode layers are solidified. Most of the prepared Bucky Gel are sheets. In 2008, Kamamichi first presented the ALM technology for Bucky Gel, where an electrode base materials-electrode structure is solidified point by point accumulatively. In this way, arbitrary shapes of Bucky Gel can be prepared (Norihiro et al., 2010). They produced a hand-shape Bucky Gel by ALM (Figure 7).

\subsubsection{DE intelligent materials}

The traditional DE actuator is a sandwich structure. It is a sort of dielectric elastic membrane material surface coated with a flexible electrode. When the voltage $U$ is applied, the top and bottom surfaces of DE materials accumulate positive and negative charges $\pm \mathrm{Q}$ due to polarization. The positive and

Figure 6 Freeform-fabricated five-layer IPMC

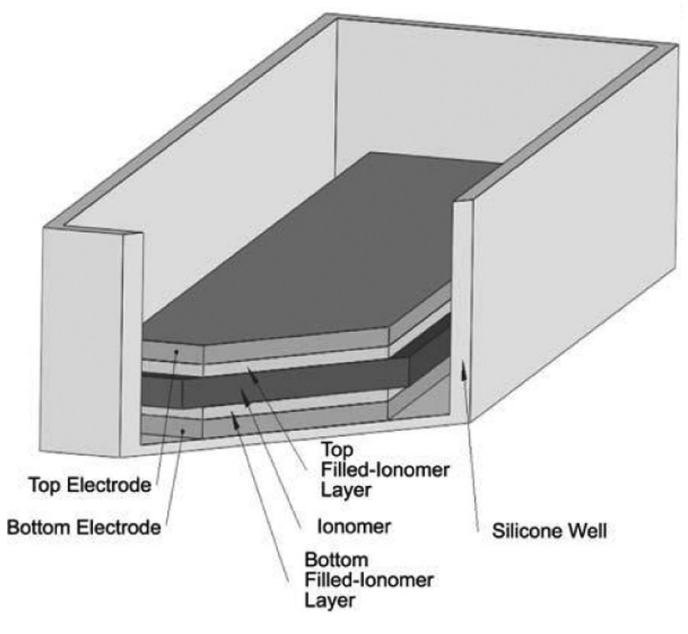

(a)

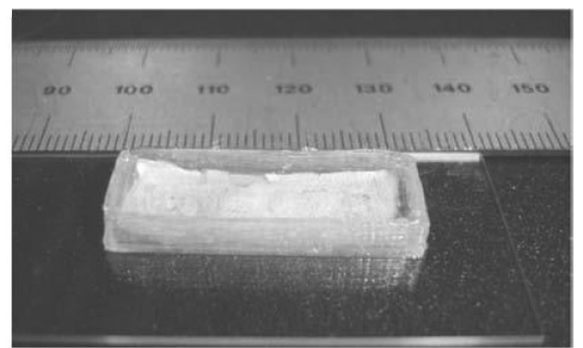

(b)

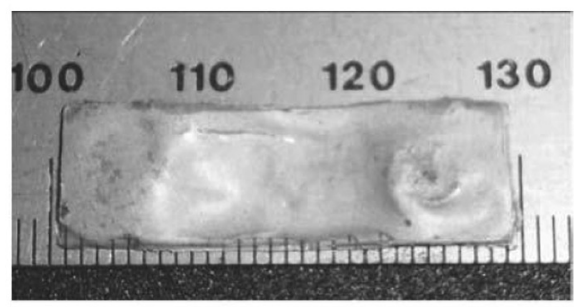

(c)

Notes: (a) Cutaway of CAD model showing layer sequence; (b) IPMC in its well; (c) after hydration and removal

Source: Malone and Hod (2006) 
Xin Li, Fianzhong Shang and Zhuo Wang

Figure 7 Sequential photographs of the printing of a hand shape

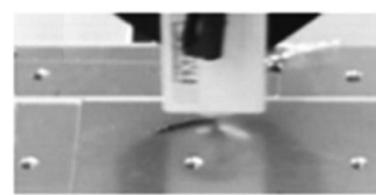

$\downarrow$

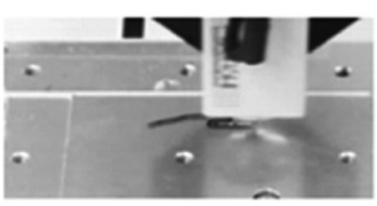

$\downarrow$

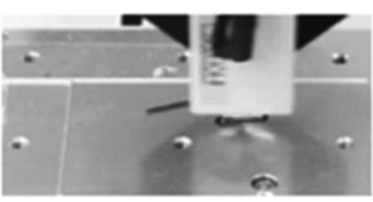

$\downarrow$

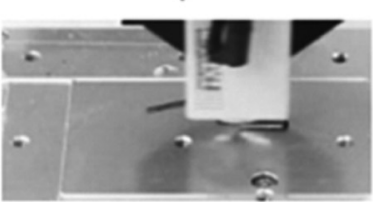

$\Downarrow$

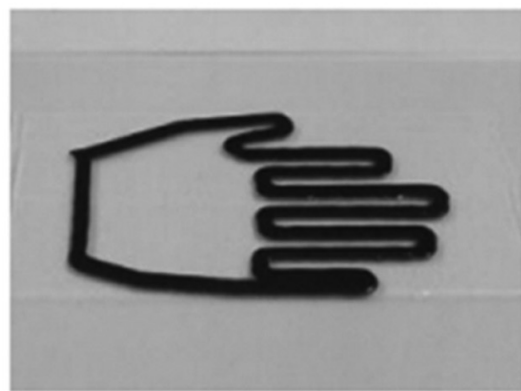

Source: Norihiro et al. (2010)

negative charges attract each other, so that a static Coulomb force is produced. So, in the thickness direction, material is compressed and its thickness decreases, which leads to expansion of area. Most of traditional DE materials are film-like.

In 2008, Rossiter and Peter (2009) first presented the ALM technology for DE. They use a polyacrylic acid material as the basement membrane material of DE. And a double-nozzle stereolithography 3D printer is adapted. One of the nozzles is used to make the supporting structure for polyacrylic acid solidification, and the other one is used to extrude liquid polyacrylic acid material, which can be cured by UV light. The formed 3D polyacrylic acid matrix material is shown in Figure 8. Then the supporting material is removed, and the surface of polyacrylic acid matrix material is coated with a flexible electrode material. Finally, the DE material is obtained.
Volume $37 \cdot$ Number $2 \cdot 2017 \cdot 170-185$

Figure 8 Fabrication of soft dielectric elastomer material using a $3 \mathrm{D}$ printing process
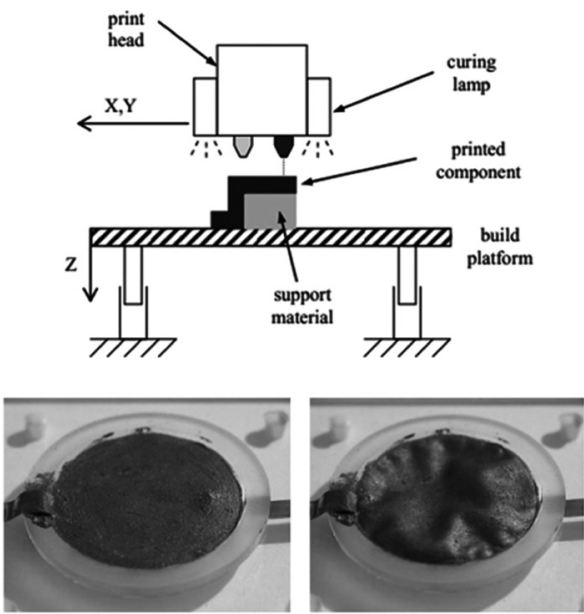

Source: Rossiter and Peter (2009)

Landgraf (2013) developed an Aerosol jet printing technology for DE. The matrix material is made of silicone. And the electrode material is made of the mixture of silicone and carbon nanotube. Sandwich structure of the DE material was realized by curing electrodematrix-electrode layer by layer. They use ultrasonic wave or pressure to change silicone liquid into aerosols. Based on that, the printing of silicone is achieved by spraying the aerosols on the surface of a working platform via the nozzle (Figure 9). Because the selected silicone is a double-component mixed curing-type silicone, to prevent the two components from mixing in the nozzle, which will cause the nozzle blocking, Landgraf (2013) designed a double-nozzle printing device. Through the two nozzles, the silicone is printed in two groups, and they are cured once in contact. So, the ALM of DE material is realized.

R. Shepherd and S. Robinson et al. proposed using UV-curable silicone 3D printing technology to fabricate the DE material (http://cornell.flintbox.com/public/project/ 24297). The matrix material is made of a kind of silicone material, which can be cured by UV light, and the electrode material is a hydrogel material mixed with conductive particles such as carbon black. The printability of silicone can be enhanced by changing its viscosity. Because the DE material prepared by ALM is not pre-stretched, the

Figure $93 \mathrm{D}$ printing of DE via aerosol spraying

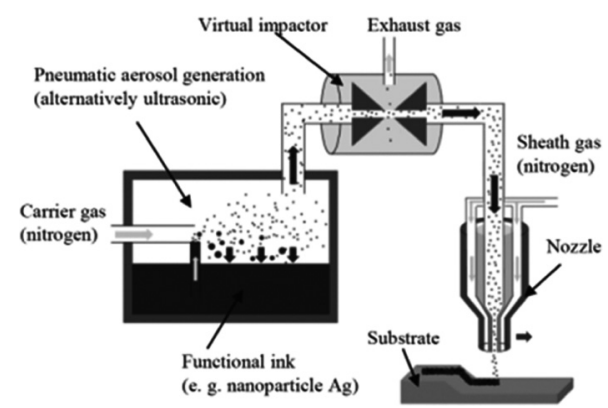


deformation of the DE material is small. However, this method makes it possible to fabricate the structure of complex DE intelligent materials.

In 2014, Creegan and Anderson presented an ALM technology of curing the DE matrix material and the DE electrode material simultaneously by UV (Figure 10).

The ALM technology for the DE material is still in the initial stages of development. Although there is a gap between the DE material prepared by the ALM technology and by the traditional method, the ALM technology makes it possible to fabricate any complex 3D DE intelligent structure in the future, which solves the problem that the traditional method cannot solve.

\section{Applications}

\subsection{Self-assembly and self-folding structures}

In April 2014, Skylar Tibbits of the Massachusetts Institute of Technology showed a combination of 3D printed plastic with an "intelligent material" that self-assembles in water. Tibbits refers to this as "4D printing" (Walton, 2013). The core of 4D printing is the Connex multi-material technology.

With Connex multi-material technology, a single print, with multi-material features, can transform from any 1D strand into $3 \mathrm{D}$ shape, a $2 \mathrm{D}$ surface into $3 \mathrm{D}$ shape or morph from one $3 \mathrm{D}$ shape into another. The Connex multi-material technology allows the researchers to program different material properties into each of the various particles of the designed geometry and harness the different water-absorbing properties of the materials to activate the self-assembly process. With water as its activation energy, this technique promises new possibilities for embedding programmability and simple decision-making into nonelectronic-based materials.

An example of $4 \mathrm{D}$ printed objects that were preprogrammed to respond to a stimulus - water - and change into other shapes is shown in Figure 11. The top figure shows a $1 \mathrm{D}$ object morphed into a $2 \mathrm{D}$ object - when inserted into water, the snake-like object forms the letters "MIT". The two figures show how one can also create self-folding cubes from both flat and wireframe structures. Other applications in Figure 12 show a single strand that self-transforms from the letters "MIT" into the letters "SAL", a flat surface that self-folds into a truncated octahedron and a flat disc that self-folds into a curved-crease origami structure. Skylar along with Stratasys Ltd. and Autodesk, Inc. using Stratasys' Connex multi-material printer and a new polymer developed to expand 150 per cent when submerged in water conducted these experiments. A new application was

Figure 10 UV ALM technology of curing DE matrix material and DE electrode material

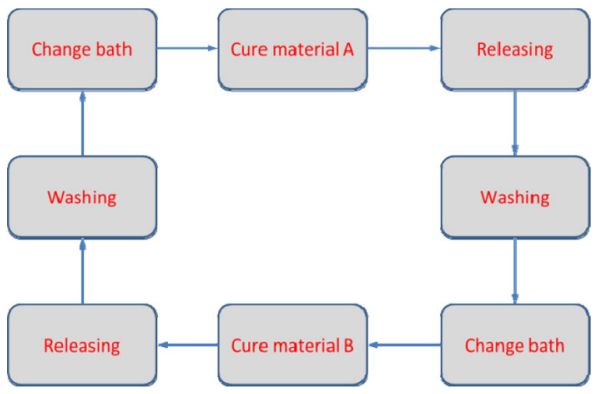

Figure 11 A single strand that self-folds into the letters "MIT" a flat surface that self-folds into a closed cube
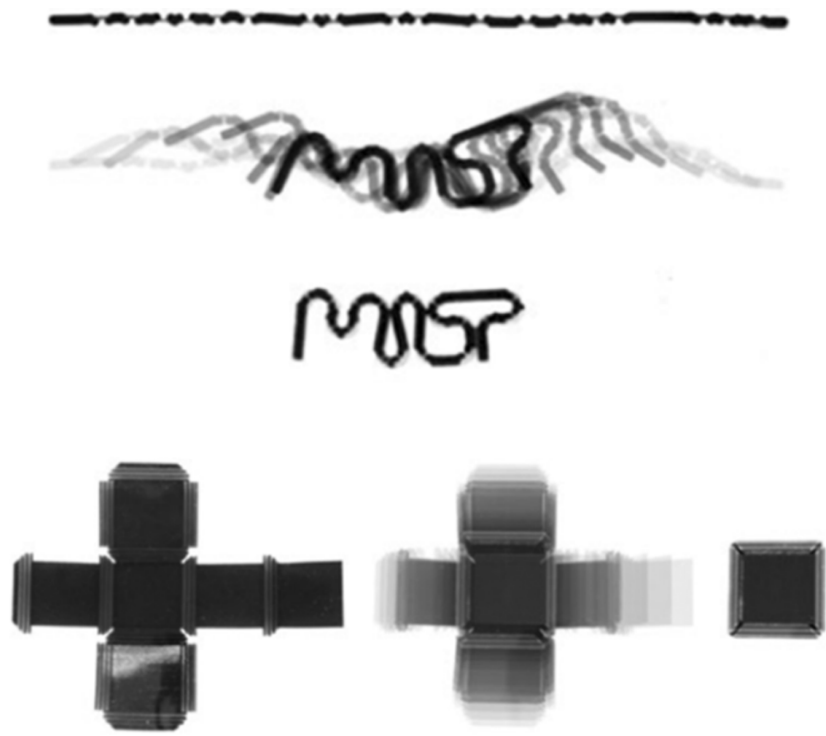

Figure $12 \mathrm{~A}$ single strand that self-transforms from the letters "MIT" into the letters "SAL" (self-assembly lab), a flat surface that self-folds into a truncated octahedron, and a flat disc that self-folds into a curved-crease origami saddle structure

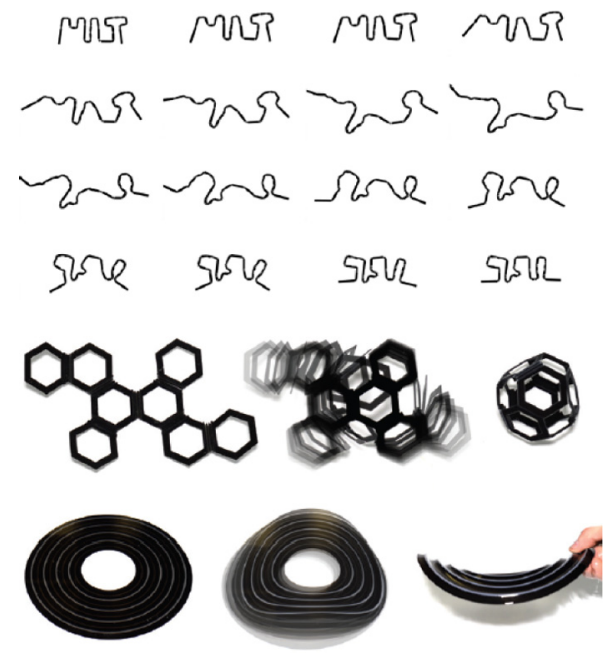

embedded into the Autodesk software, Project Cyborg, to simulate the dynamics of $4 \mathrm{D}$ printed objects and their material optimization. This technology has attracted substantial press attention around its potential for manufacturing (Tibbits, 2014). Another 4D printing technology involves embedding wiring or conducting parts into special compliant components during the $3 \mathrm{D}$ printing job. After the object is printed, the parts can be activated by an external signal to trigger full assembly actuation (Figures 13 and 14). This approach has potential implications for areas such as robotics, furniture and building construction.

Other 4D printing approaches include composite materials that can morph into several different complicated shapes based on a different physical mechanism and heat activation (Ge et al., 2013). Also, demonstrations have been 
Figure 13 Robotic finger designed and created via in situ embedding with an AM process

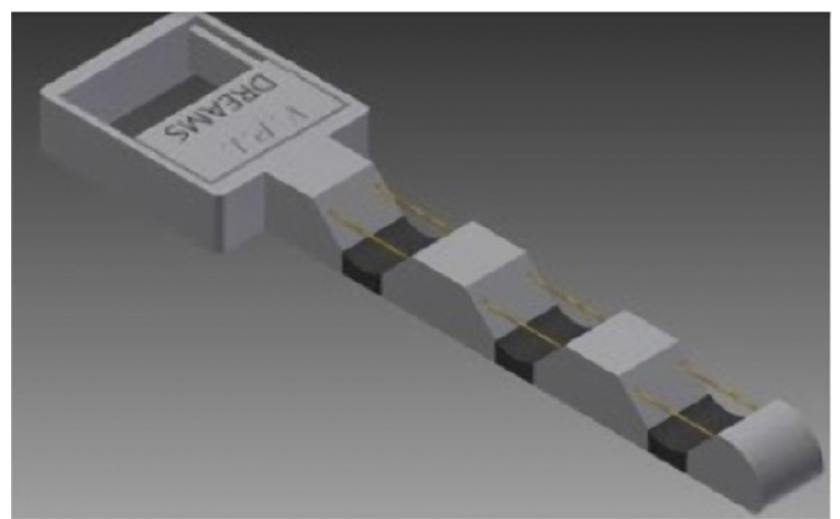

(a)

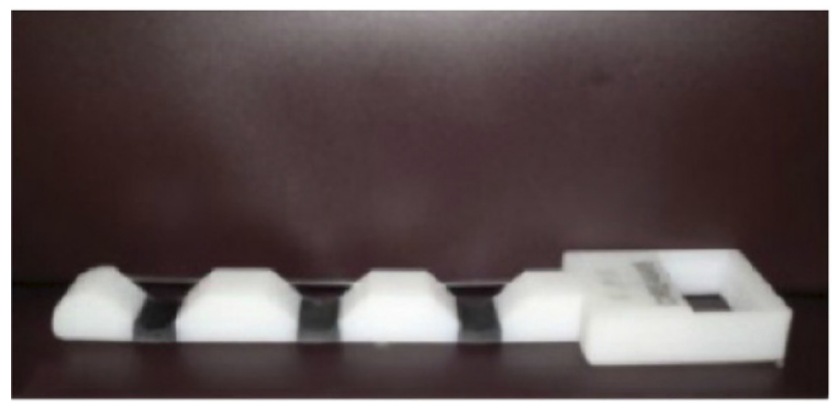

(b)

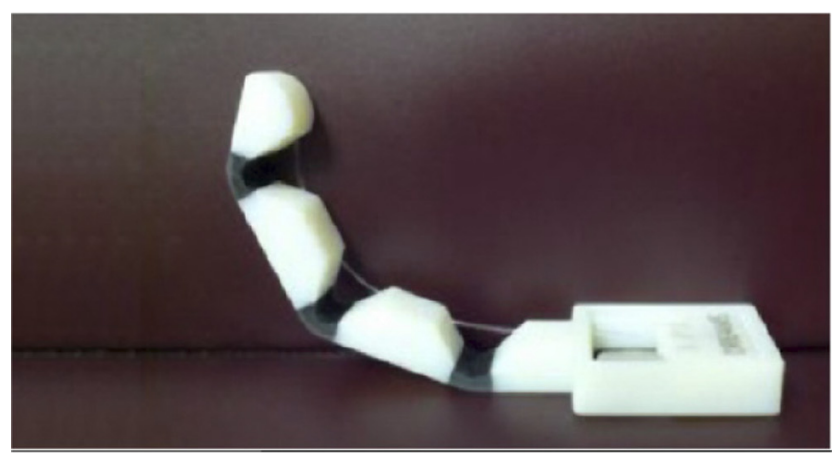

(c)

Notes: (a) Computer-aided design (CAD) representation; (b) finger as built (with embedded monofilament fiber); (c) finger actuated via a sliding joint

Source: Justin et al. (2011)

made of materials that self-fold because of light exposure (Ying et al., 2012).

Developed an ALM technology for SMG, which is currently used in the manufacture of smart medical bandage, zoom lens and bionic robot, etc.

Raised an ALM technology for SMP, and used this to fabricate intelligent structures that have self-assembly and self-folding functions (Tolley et al., 2013; Felton et al., 2015). First, the SMP is accumulated to a rigid substrate board. After solidification, the SMP and the rigid substrate board are tightly combined to a plane structure. Then,
Figure 14 Integrated sensing and actuating wing flap manufactured via a materials jetting process
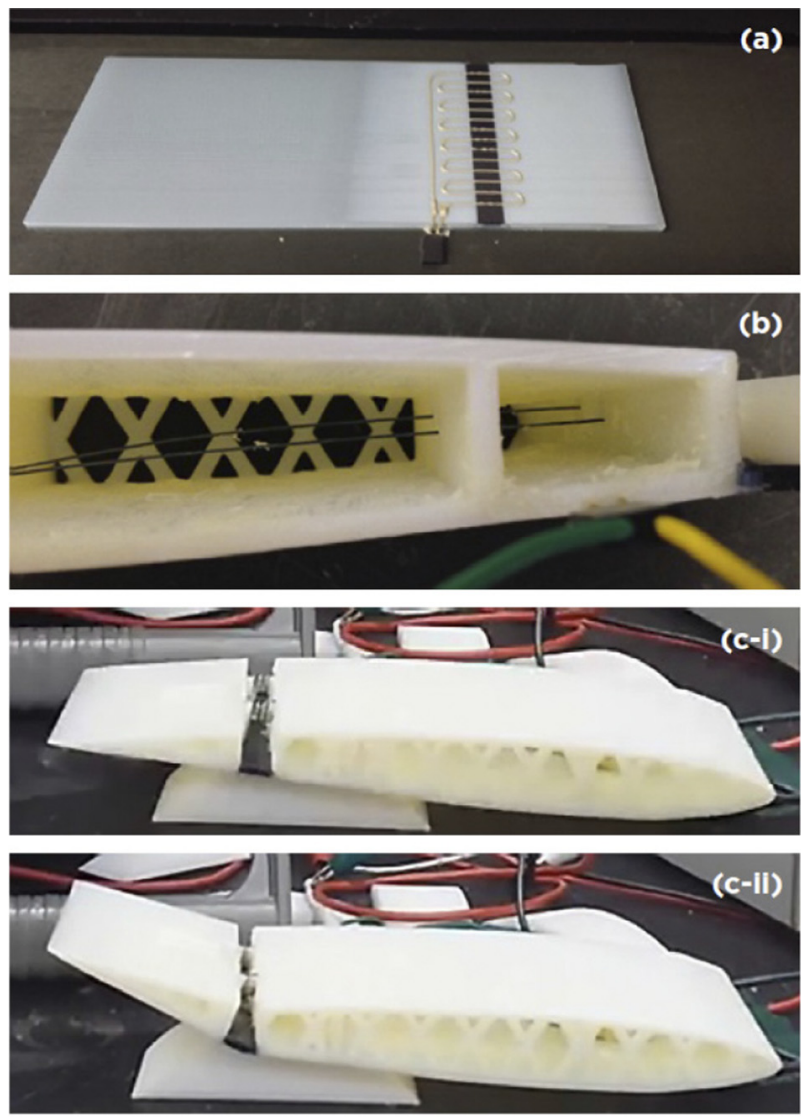

Notes: (a) Embedding plane and geometry of directly written conductor shown on build tray during the mid-print deposition step (Du Pont 5021 conductive silver ink; conducting lines are $700 \mu \mathrm{m}$ in width and $32 \mu \mathrm{m}$ in height); (b) detailed view of embedded, actuating SMA wire within final printed geometry; (c) integrated sensing and actuating wing flap (i) before and (ii) after electrical current application. Wing is 7 in length, 2.5 epth and 0.75 in height at its widest point.

Source: With permission from Dr Christopher B. Williams, DREAMS Laboratory, Virginia Tech; www.dreams.me.vt.edu

under external stimulus such as light, temperature, current, etc., the SMP generates a volume expansion or contraction, which makes the plane structure become a $3 \mathrm{D}$ structure (Figure 15).

\subsection{Active composites structures}

Ge et al. (2013) proposed to implement a 4D printing technology by using multi-material ALM technology. In their method, the SMP fiber is combined with an organic polymer matrix by printing them simultaneously. With the result that the structure they make can be changed with time. Figure 16 shows the printing process of the SMP fiber and organic polymer matrix. Figure $16(\mathrm{a})$ is the schematic showing the printing process of printed active composite materials. The inkjet heads move horizontally above the tray depositing multi-material droplets of polymer ink at prescribed positions, wiping them into a smooth film, and then UV photo 
Figure 15 (a) A flat composite programmed to fold into a bumblebee-like structure; (b) the bumblebee after folding; and (c) the bumblebee with its wings raised

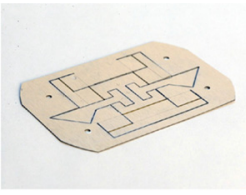

(a)

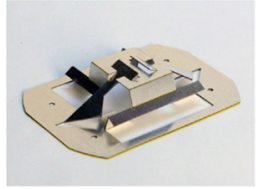

(b)

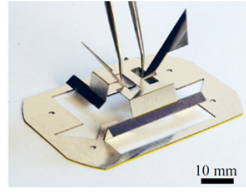

(c)
Source: Felton et al. (2015)

polymerizing the film. After one film layer is completed, the tray moves down to print the next layer. Figure 16(b) is the schematic of a printed active composites (PAC) lamina. Fibers are oriented at an angle $\theta$ from the $x$-direction (the loading direction).

If they combine the intelligent structure in Figure 16 with another organic polymer layer to form a double-layered structure, by changing the temperature, the transformation of bending deformation and initial shape can be realized. And the bending deformation amplitude of the intelligent structure can be changed by changing the direction of the SMP fiber. So, the deformation of the structure is controlled. Figure 17 shows the anisotropic shape memory behavior of the active lamina to create PAC laminates.

Recently, Malone and Hod (2006) selected an IPMC from the literature and their own preliminary experiments as most promising for freeform fabrication. They performed material formulation and manual device fabrication experiments to arrive at materials that are amenable to robotic deposition, and developed an ALM process that allows the production of complete IPMC actuators and their fabrication substrate integrated within other freeform-fabricated devices. Malone and Hod (2006) freeform-fabricated simple IPMCs, explored some materials/performance interactions and preliminarily characterized these devices in comparison to devices produced by traditional methods.

In their research, room temperature vulcanization (RTV) silicone was selected as a containment material. They routinely achieve $250-\mathrm{mm}$ resolution when fabricating structures with it, and deposit it with good compatibility on most of the other materials that their system uses. Rectangular silicone wells [Figure 18(a)]
Figure 17 Complex low-temperature shapes of active composite laminates obtained by design of the laminate architecture

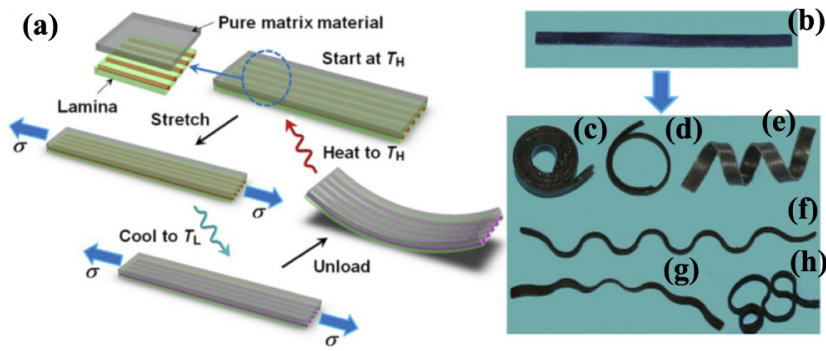

Notes: (a) A two-layer laminate designed with one layer being a lamina with fibers at a prescribed orientation and one layer being pure matrix material is printed, then heated, stretched, cooled and released. Upon release of the stress, it assumes a complex shape, depending on the laminate architecture. When reheating, it then assumes its original shape, a flat rectangular strip; (b) an actual strip in its original shape; (c)-(h) the results of this process with differing fiber architectures

Figure 18 Fabricated rectangular silicone wells

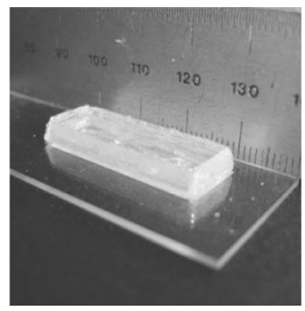

(a)
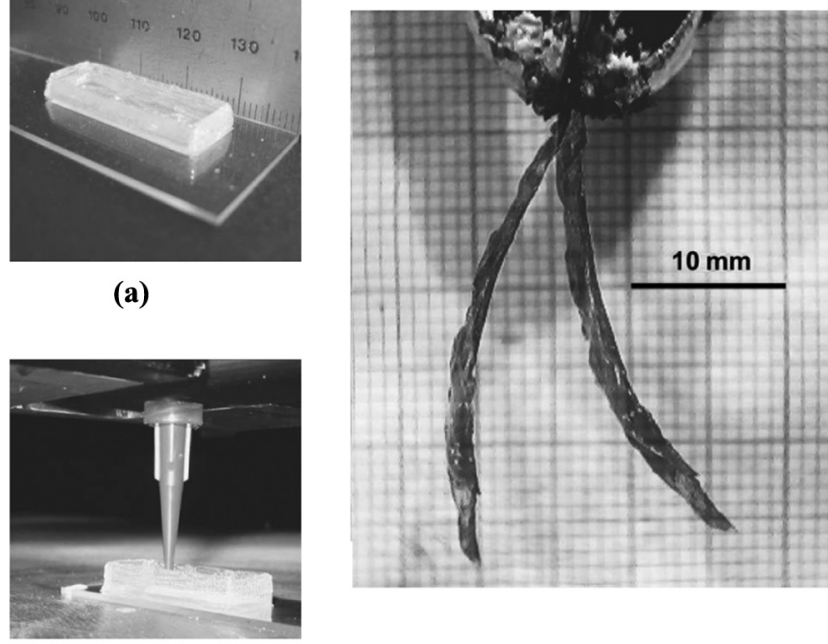

(b)

(c)

Figure 16 Schematic illustrating the PAC printing process

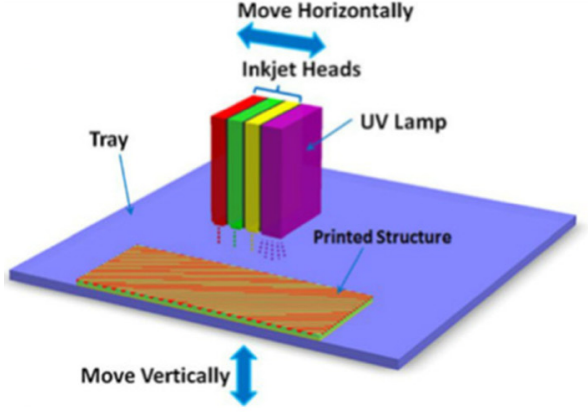

(a)

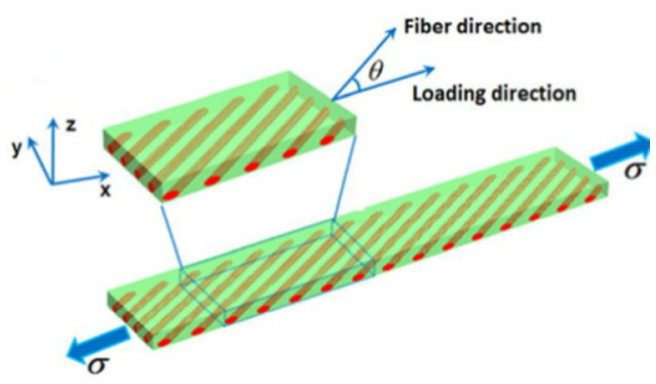

(b) 
with $1 \mathrm{~cm} \times 3 \mathrm{~cm} \times 2.5 \mathrm{~mm}$ internal dimensions were deposited onto glass microscope slides for use as containers for IPMC fabrication. When cast into these wells, they observed that there is a tendency for the ionomer to seep under the lower electrode material, and also to be drawn toward the boundaries of the well. This apparently produces stresses in the film as it dries, leading to cracking. With the addition of 33 Wt. \% of DMF (N-N-dimethylformamide) to the ionomer, the cracking problem was eliminated at the cost of greatly increased drying time. Carbon black powder is used to help suspend the silver powder in the electrode material to improve dispensability. This formulation, dubbed electrode material 6 (EM6), can be dispensed from a 0.5-mm ID syringe needle without clogging. A four-probe measurement technique was used to obtain the resistivity of this material; the values for pure silver, carbon and platinum are provided for reference.

To be able to deposit liquid materials precisely into the silicone well, Malone and Hod (2006) extended the manufacturing planning software of their fabrication system to allow exceptions to strict layered manufacturing - a technique they call "backfill deposition". Using backfill deposition, the silicone well can be assigned a higher fabrication priority than the materials to be deposited into it, and it will be completely fabricated to its full height before the deposition of the first electrode layer begins [Figure 18(b)]. This greatly improves the speed of fabrication by reducing the number of material changes, and the quality of cast materials by allowing the deposition nozzle to be at its optimal height above the layer beneath. They deposited the reformulated materials with their freeform fabrication system to produce several working IPMC devices. The actuation of these devices was verified qualitatively by videography [Figure 18(c)]. The devices suffer from internal shorting, and have a very short service life in air - typically only two or three actuation cycles. Nevertheless, these are the first completely freeform-fabricated IPMC actuators.

To enable quantitative testing of the performance of the IPMC devices being produced, they developed a test apparatus (Figure 19) that interfaces with a PC-based data acquisition system (IOTech DAQBoard 2000, running DASYLab DAQ software).

Associated with the change of a cation in the liquid Nafion from $\mathrm{H}^{+}$to $\mathrm{Li}^{+}$, they observe a performance increase in output shear stress for five-layer devices of almost 3.5 times - even greater than expected. Note that while there is a performance gap of two orders of magnitude between the best freeform-fabricated device and the best published results for IPMC's made with commercial Nafion membranes, the published performance for devices made with Nafion cast from the liquid dispersion is only superior by a factor of two, despite using the $\mathrm{Pt}$ salt electroding process applied in the best published results. This suggests that the dispersion-cast membrane is somehow inferior for actuation applications.

\subsection{Environmental adaptive mechanism and structural health monitoring}

A lot of intelligent material also has driving function and sensing function, such as SMA. It not only can be used as an actuator to produce deformation under different temperatures but also can measure the strain, temperature and crack inside the structure in real time, detecting the fatigue and damage.

Proposed the ultrasonic additive manufacturing (UAM) technology to combine different metal materials and intelligent materials into intelligent structures. This kind of intelligent structure has the function of shape changing under different circumstances and the function of structure monitoring (http://spie.org/x105826.xml). UAM refers to combining metal foils with the ultrasonic wave at room temperature, so that $3 \mathrm{D}$ solid structures can be realized layer by layer. Use the UAM technology to combine the SMA and other intelligent materials into the metal matrix, and the shape of obtained intelligent structure can be changed according to different demands (Figure 20). Also, this technology can realize the health monitoring and life prediction of intelligent structure. At present, the intelligent structure can be used in the design and manufacture of intelligent vehicles and intelligent aerocrafts.

Recent multiscale models developed had a simplified framework with three scales: structural, material macrostructure and material microstructure. Previously, there was insufficient modeling at the microstructure level. Therefore, they used a bottom-up approach starting at the microstructure. At that scale, a single alumina fiber (used for reinforcement) and surrounding aluminum matrix created a representative volume element (RVE) that they used as a building block for describing a UAM composite. They created

Figure 19 Schematic of test apparatus for acquiring force output, voltage and current versus time

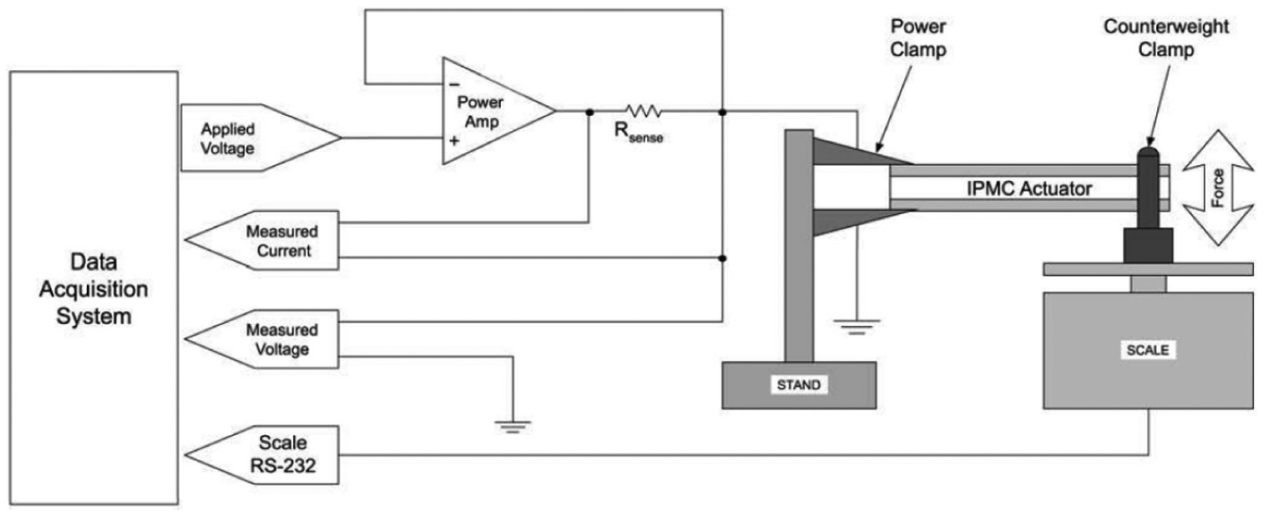


Figure 20 Aluminum hinge actuated by shape memory (nickel titanium) wires
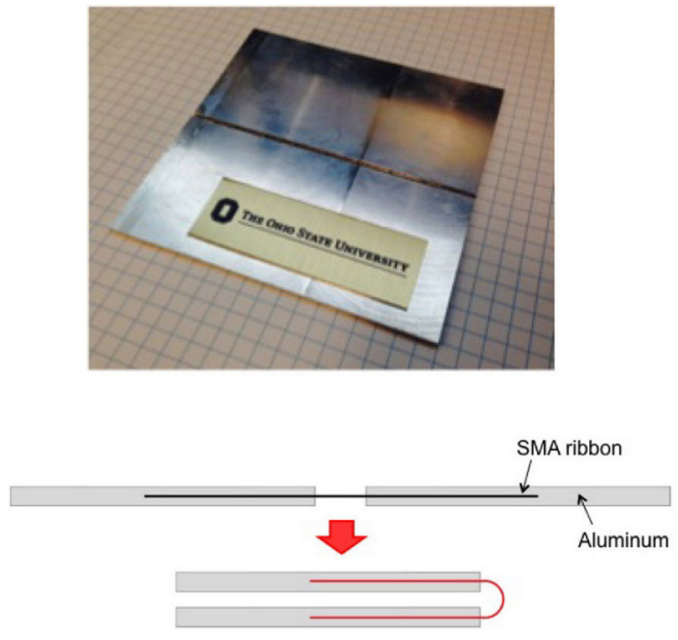

the macrostructure scale, defined as a single foil layer, by periodic assembly of RVEs, considering periodic boundary conditions. At the structural scale, they combined several tapes to create a composite. At this level, macroscopic material properties are defined and the interactions between the composite and UAM process take place. They implemented these scales in COMSOL Multi-Physics, a commercial finite element analysis solver. They applied compressive and shear loads typical of very-high-power UAM and modeled surface roughness effects and different fiber geometries. They found that surface roughness (as transferred to the tape from the sonotrode's texture) had a significant effect on bonding, with large asperities, inducing greater plastic deformation and therefore improved bonding (Figure 21).

Have conducted a design of experiments' analysis on UAM builds fabricated under various treatment combinations and mechanically tested in shear and multiaxial tension. Work on aluminum-aluminum and titanium-aluminum builds used a Taguchi L18 experimental design matrix that factors temperature, normal force, welding velocity and vibration amplitude (Hopkins et al., 2010, 2012), and they used analysis of

Figure 21 Plastic deformation for $6 \mu \mathrm{m}$ roughness at $13 \mu \mathrm{m}$ sonotrode deflection

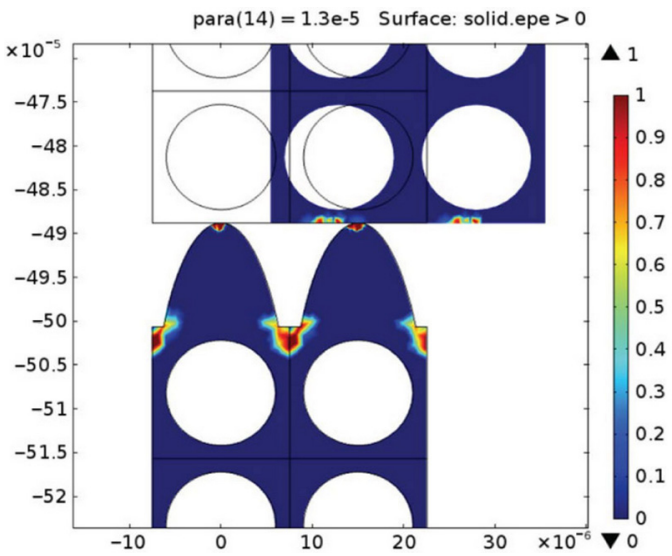

variance statistical testing to calculate trends and understand the effect of process parameters on mechanical strength.

Critical advantages of UAM for the transportation industry are reducing mass and part count, and embedding advanced features directly within a structure. To realize these benefits, research is needed to better understand the mechanisms that govern joining of dissimilar joints involving harder metals, such as stainless steel, titanium alloys (Ti6Al4V, for example) and structural aluminum (such as 6XXX and 7XXX alloys). On the applications side, their work will continue to focus on embedding features into metals, such as intelligent material sensors and actuators, electronic components and thermal management devices, among others.

\subsection{Self-deployable systems}

Tolley et al. (2013) fabricated self-deployable systems (SDSs) by ALM technology with SMP (Samuel et al., 2013). They use the ALM technology to combine the SMP material and hard matrix material into an intelligent structure, which can realize self-assembly and self-folding under external environmental stimulus. SDS can be applied to detectors, logistics, etc. (Samuel et al., 2013). One of the most famous applications in detectors is the Inchworm Robot. By controlling the repeated bending and folding of the Inchworm Robot, its progressive motion can be realized (Figure 22). Figure 22(a) is the two-dimensional inchworm robot, before it has folded into its functional shape. Figure 22(b) is the folded inchworm, after the servo and battery have been added. This robot weighs $29 \mathrm{~g}$, and moves at a rate of $2 \mathrm{~mm} / \mathrm{s}$.

To show self-deployability, a schematic is shown in Figure 23. Locomotion is achieved by angling the "feet" at the bottom of the front and back walls [Figures 23(a) and (b)) to create an asymmetric friction. The front wall fold angle relative to the robot body is $45^{\circ}$, and the back wall

Figure 22 The inchworm robot

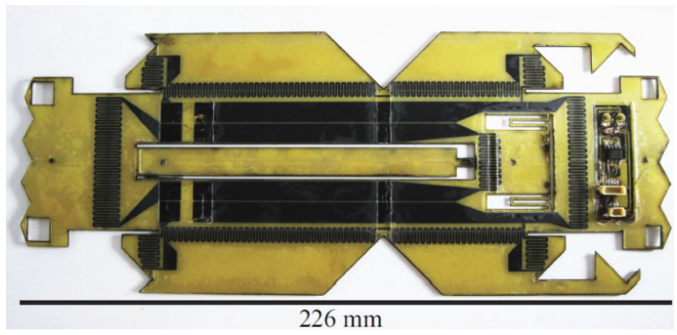

(a)

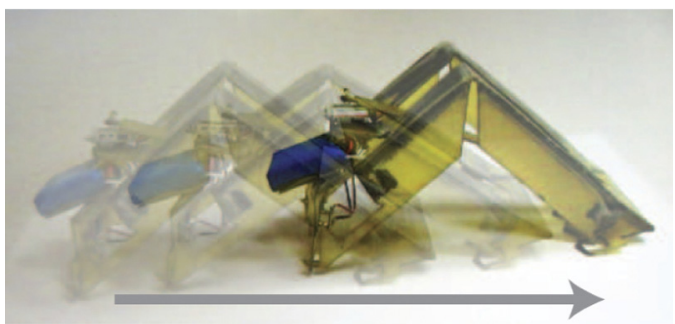

(b)

Notes: (a) The two-dimensional inchworm robot; (b) the folded inchworm 
Figure 23 The inchworm robot after the folding of its front-, backand sidewalls (a)

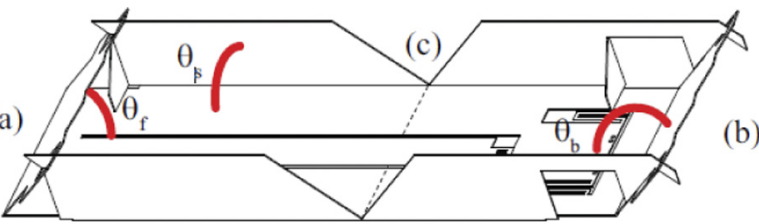

(c)

(a)

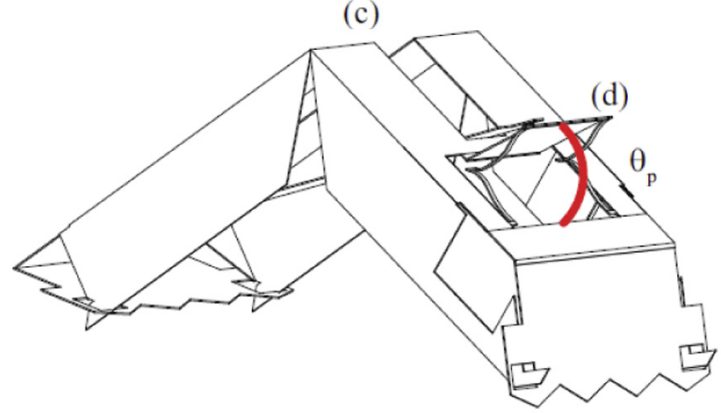

Notes: (a) A front wall and (b) back wall angled to create feet with asymmetric friction. The front wall fold angle $\theta_{\mathrm{f}}$ is $45^{\circ}$ and the back wall fold angle $\theta_{\mathrm{b}}$ is $135^{\circ}$; (c) side walls with fold angles, $\theta_{s}$, of $90^{\circ}$, and a servo platform; (d) with a fold angle, $\theta_{\mathrm{p}}$, of $45^{\circ}$

fold angle is $135^{\circ}$. Sidewalls [Figure 23(c)] are included at right angles to the robot body to improve structural rigidity. A single dynamic hinge in the middle is controlled by a slider-crank mechanism actuated by a linear servo. The servo is driven by a $0.3 \mathrm{~Hz}$ triangle wave from a microcontroller (ATtiny 13, Atmel), and powered by a $7.4 \mathrm{~V}$ lithium polymer battery (EFLB1202S20, E-flite). The servo is mounted on a platform that folds up $45^{\circ}$ from the body [Figure 23(d)].

Materials were chosen for their physical properties, cost and ease of use. A 125-mm-thick sheet of polyetheretherketone (APTIV 1,000, Victrex) is used as a passive substrate because of its high flexural strength $(163 \mathrm{MPa})$ and resistance to fatigue along folds. Pre-stretched polystyrene (KSF50-CIJ, Grafix) was chosen for the contractile layer because of its high compressive strain (50 per cent) when heated above its relatively low transition temperature $\left(160^{\circ} \mathrm{C}\right.$ ) (Ying et al., 2012). The circuits are made on a composite consisting of an $18-\mathrm{mm}$ copper layer and a $12-\mathrm{mm}$ sheet of polyimide.

After completion of the folding process and manual addition of a servo and battery, the inchworm robot weighed $29 \mathrm{~g}$ and measured $145 \mathrm{~mm}$ in its extended position. The robot was capable of moving on paper at a rate of $2 \mathrm{~mm} / \mathrm{s}$ for a $0.3 \mathrm{~Hz}$ contraction frequency, or 0.8 body lengths per minute, and consumed $0.9 \mathrm{~W}$ during locomotion. Because of the complexity of asymmetric surface friction, this speed was irregular (Figure 24). The stroke length of the foot displacement measured $10 \mathrm{~mm}$; therefore, only 20 per cent of the motion was converted into locomotion, and the rest was lost to slippage. The contraction frequency was chosen because higher frequencies resulted in even more slippage.
Figure 24 The displacement of the front edge of the inchworm robot over time

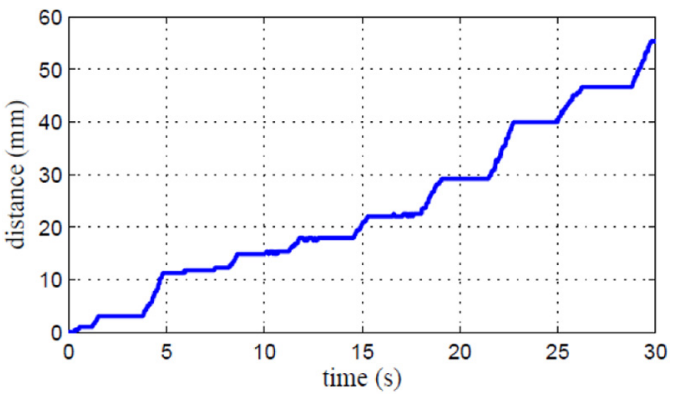

\section{Arm-type ALM technology of intelligent materials based on thermosetting polyurethane}

The research group of Professor Jianzhong Shang in National University of Defense Technology (NUDT) in China has proposed an arm-type ALM technology of SMP based on thermosetting polyurethane. This technology is suitable for large-scale intelligent structure manufacturing because of its fast curing. However, this technology is in the early research period, and large-scale structure manufacturing has not been applied.

The thermosetting polyurethane is a matrix material for SMP. The mixture material has a better toughness than single SMP material, and the thermosetting polyurethane has a good chemical stability, which manifests as good corrosion resistance and good thermostability. Hence, the joining of the thermosetting polyurethane will not influence the SME of the fabricated structure under external stimulus. After conducting a large number of material experiments with different polyurethane, the use of very rapidly hardening two-component thermosetting polyurethane were selected as the more appropriate mix of materials and first prototypes of the extruder were created. Table III is the recipe. Component A is a prepolymer of $20 \mathrm{~g}$ pure methylene diphenyl diisocyanate (MDI) and the mixture of $194.03 \mathrm{~g}$ polypropyleneglycol (PPG) and $0.97 \mathrm{~g} \mathrm{1,2-butanediol} \mathrm{(BD),}$ while Component $\mathrm{B}$ is a prepolymer of $80 \mathrm{~g} \mathrm{MDI}$ ( 80 per cent) and a mixture of $134.32 \mathrm{~g}$ PPG and $0.68 \mathrm{~g} \mathrm{BD}$. In the components, MDI could provide isocyanate groups (-NCO) for the polyurethane, and PPG and BD could provide hydroxyl $(-\mathrm{OH})$ for the polyurethane. After a large number of calculations and experiments, this recipe of Component $\mathrm{A}$ (prepolymer of isocyanate) and Component B (prepolymer of polyether polyol) is selected. With this recipe, after the $1: 1$ mixture, the fast-curing polyurethane can reach a curing rate of 90 per cent after $10 \mathrm{~s}$. A constant-rate plunger extruder is used to mix the source material components. Both source material components are pushed through the mixer with such speed that solidification takes place precisely $2 \mathrm{~mm}$ away from

Table III The recipe of the two-component thermosetting polyurethane

\begin{tabular}{ll}
\hline Component A & Prepolymer of $20 \mathrm{~g}$ pure MDI; \\
& Mixture: $194.03 \mathrm{~g} \mathrm{PPG}$ and $0.97 \mathrm{~g} \mathrm{BD}$ \\
Component B & Prepolymer of $80 \mathrm{~g} \mathrm{MDI}(80 \%) ;$
\end{tabular}
Mixture: $134.32 \mathrm{~g}$ PPG and $0.68 \mathrm{~g} \mathrm{BD}$ 
the nozzle aperture (Figure 25). After exhaustive tests of the material properties of the mixed thermosetting polyurethane in different heating and deposition speeds, the most optimized scenario was $350^{\circ} \mathrm{C}$ and $4 \mathrm{~mm} / \mathrm{s}$.

A fabricated structure by the arm-type ALM technology of SMP based on thermosetting polyurethane is shown in Figure 26, and this structure can change its shape by raising the temperature to its shape memory transition temperature, $\mathrm{T}_{\text {trans }}$. Our research shows that when the content of thermosetting polyurethane increases, its tensile strength and breaking elongation increase, as well as the shape-memory transition temperature $T_{\text {trans }}$. While, when the content of SMP increases, its deformation recovery rate increases, and the recovery time is reduced slightly. When the content of SMP reaches its "limit", it only takes $12 \mathrm{~s}$ to obtain a deformation recovery rate of 99 per cent.

SMP material with thermosetting polyurethane matrix material could overcome low mechanical strength and small deformation recovery force of single-phase SMP. Compared to other thermosetting polymers, thermosetting polyurethane has the advantages of high heat-resistance temperature, big deformation recovery force and low cost. It will have an important application prospect in space-deployable structure and morphing aircraft wing, etc.

However, in the experiments, the foaming of polyurethane material will affect the accuracy of the printed structure, namely, the printing precision control is very difficult because of the foaming of the polyurethane. Therefore, in the following study, the foaming of polyurethane must be "inhibited" to improve the printing precision. Because the foaming of polyurethane is

Figure 25 Arm-type ALM device of SMP with thermosetting polyurethane

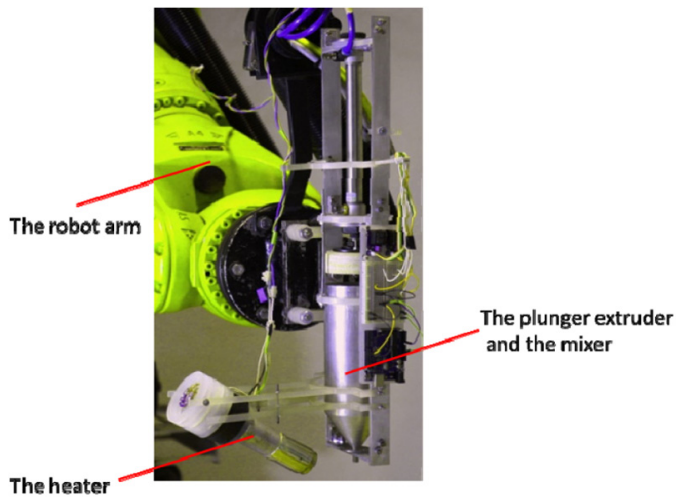

Figure 26 A fabricated structure in SMP with thermosetting polyurethane matrix material and the SME

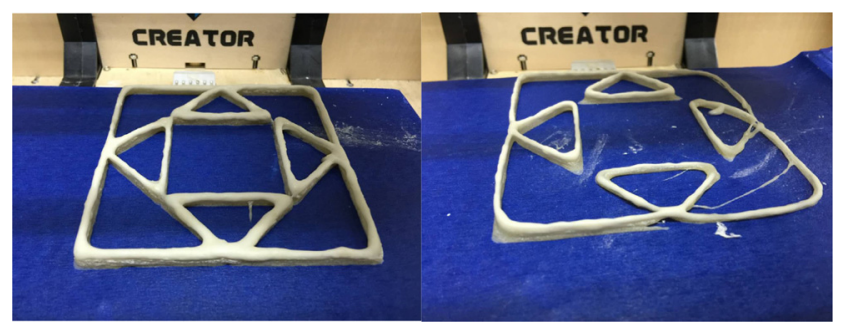

inevitable in the process of reaction, the "inhibited" strategies should be:

- make the pre-polymerization of Components A and B to be fully reacted, so that the bubbles could be tiny and uniformly distributed; and

- determine the minimal size of printing so that after expansion, the produced part would be in its correct size and dimension; in other words, the expansion ratio before printing should be analyzed and controlled.

\section{Conclusion}

In this paper, recent advanced research studies of the ALM technology of intelligent materials and the development of the novel 4D printing technology are first reviewed. Then, the applications of ALM technology of intelligent materials in the field of environmental adaptive mechanism, structural health monitoring, flexible mechanism and self-deployable systems are introduced. At last, we provide a brief outline of our research on an arm-type ALM technology of SMP with thermosetting polyurethane.

4D printing technology and its application in intelligent material structure are still in its initial stage. However, its research and development applications will have a profound impact on the traditional mechanical structure design and manufacturing. This trend is reflected in the following aspects: - 4D printing intelligent material will change the past "mechanical transmission + motor drive" mode to the in situ drive mode of intelligent material, which is no longer restricted by the DOFs of mechanical structure. So, it can achieve the controlling function of DOFs and stiffness, and the weight will be significantly reduced.

- With 4D printing technology, intelligent material structures integrated with drivers and sensors can be produced. EAP material has good driving and sensing performance, that is, it can generate deformation under the action of electric field, and, in the meantime, voltage and current signal can be the outputs. Our group combined SMP material with thermosetting polyurethane to improve its toughness. Also, it will not influence the SME of SMP materials because it has good chemical stability after shaping. And an arm-type ALM device for the mixture is designed. In the future, intelligent materials mixed with different functional polymer will be developed, and these sorts of materials will be more suitable for 4D Printing.

- A variety of intelligent materials for $4 \mathrm{D}$ printing technology will be researched and developed, and the response to different external stimulus will be more diverse. At present, the incentive mode and the deformation mode of the intelligent materials for 4D printing are limited. Tibbits et al. are developing a $4 \mathrm{D}$ printing technology of intelligent material that can respond to vibration and sonic wave.

\section{References}

Aissa, B., Therriault, D., Haddad, E. and Jamroz, W. (2012), "Self-healing materials systems: overview of major approaches and recent developed technologies", Advances in Materials Science and Engineering, Vol. 17, pp. 203-212.

Bar, C. and Yoseph, F. (1998), "Low-mass muscle actuators using electroactive polymers (EAP)", The 5th Annual 
International Symposium on Smart Structures and Materials, Lyon, France, pp. 1346-1352.

Bogue, R. (2013), "3D printing: the dawn of a new era in manufacturing", Assembly Automation, Vol. 33, pp. 307-311.

Bose, S., Vahabzadeh, S. and Bandyopadhyay, A. (2013), "Bone tissue engineering using 3D printing", Materials Today, Vol. 16, pp. 496-504.

Carreño-Morelli, E., Martinerie, S. and Bidaux, J.E. (2007), "Three-dimensional printing of shape memory alloys", Materials Science Forum, Vol. 25, pp. 477-480.

Chen, H., Wang, Y. and Sheng, J. (2013), "Research of electro-active polymer and its application in actuators", Fournal of Mechanical Engineering, Vol. 49, pp. 205-214.

Eujin, P. (2014a), “4D printing - revolution or fad", Assembly Automation, Vol. 34, pp. 123-127.

Eujin, P. (2014b), "4D Printing: dawn of an emerging technology cycle", Assembly Automation, Vol. 34, pp. 310-314.

Felton, S.M., Becker, K.P. and Aukes, D.M., (2015), "Self-folding with shape memory composites at the millimeter scale", Fournal of Micromechanics and Microengineering, Vol. 25, pp. 1-8.

Frazier, W.E. (2014), "Metal additive manufacturing: a review", Fournal of Materials Engineering and Performance, Vol. 23, pp. 1917-1928.

Fulcher, J.T., Lu, Y.C., Tandon, G.P. and Foster, D.C. (2010), "Thermomechanical characterization of environmentally conditioned shape memory polymer using nanoindentation", Proceedings of SPIE, Vol. 76, pp 4-40.

Ge, Q., Dunn, C. and Qi, H. (2014), “Active origami by 4D printing”, Smart Materials and Structures, Vol. 23, pp. 187-197.

Ge, Q., Qi, H. and Dunn, M. (2013), "Active materials by four-dimension printing", Applied Physics Letters, Vol. 103, pp. 1-5.

Holmes, T., Delacalle, S., Su, X., Rich, A. and Zhang, S. (2010), "Extensive neurite outgrowth and active neuronal synapses on peptide scaffolds", Proceedings of the National Academy of Sciences, Vol. 97, pp. 6728-6733.

Hopkins, C., Dapino, M. and Fernandez, S. (2010), "Statistical characterization of ultrasonic additive manufacturing Ti/Al composites", ASME fournal of Engineering and Material Technology, Vol. 132, pp. 41-47.

Hopkins, C., Wolcott, P. and Dapino, M. (2012), "Optimizing ultrasonic additive manufactured Al 3003 properties with statistical modeling”, ASME fournal of Engineering and Material Technology, Vol. 134, pp. 11-15.

http://cornell.flintbox.com/public/project/24297

http://spie.org/x105826.xml

Huang, W., Song, C. and Fu, Y., (2013), "Shaping tissue with shape memory materials", Advanced Drug Delivery Reviews, Vol. 65, pp. 515-535.

Huang, W., Yang, B. and Zhao, Y. (2010), "Thermomoisture responsive polyurethane shape-memory polymer and composites: a review", fournal of Materials Chemistry, Vol. 20, pp. 3367-3381.

Huang, W., Zhao, Y. and Wang, C. (2012), "Thermo/ chemoresponsive shape memory effect in polymers: a sketch of working mechanisms, fundamentals and optimization", Fournal of Polymer Research, Vol. 19, p. 9952.
Ivanova, O., Williams, C. and Campbell, T. (2013), “Additive manufacturing (AM) and nanotechnology: promises and challenges", Rapid Prototyping fournal, Vol. 19, pp. 353-364.

Jiang, H.Y., Kelch, S. and Lendlein, A. (2006), "Polymers move in response to light", Advanced Materials, Vol. 18, pp. 1471-1475.

Justin, L., Amelia, M. and Christopher, B. (2011), "A Method for creating actuated joints via fiber embedding in a polyjet 3D printing process", 22nd Annual International Solid Freeform Fabrication Symposium, Austin, TX.

Karl, K., Ken, M. and Takushi, S. (2014), "Impact of viscoelastic properties on Bucky-Gel actuator performance", Fournal of Intelligent Material Systems and Structures, Vol. 18, pp. 2235-2245.

Landgraf, M. (2013), "Aerosol jet printing and lightweight power electronics for dielectric elastomer actuators", The 3rd International Electric Drives Production Conference (ED-PC), IEEE, Nuremberg, pp. 824-832.

Lee, K., Koerner, H. and Vaia, R., (2011), "Light-activated shape memory of glassy, azobenzene liquid crystalline polymer networks", Soft Materials, Vol. 7, pp. 4318-4324.

Lendlein, A. (2010), Shape-Memory Polymers, Springer, Berlin.

Lendlein, A. and Kelch, S. (2005), "Shape-memory polymers as stimuli-sensitive implant materials", Clinical Hemorheology and Microcirculation, Vol. 32, pp. 105-116.

Malone, E. and Hod, L. (2006), "Freeform fabrication of ionomeric polymer-metal composite actuators", Rapid Prototyping fournal, Vol. 5, pp. 244-253.

Mondal, S. and Hu, J. (2006), "Temperature stimulating shape memory polyurethane for smart clothing", Indian Fournal of Fiber Text and Research, Vol. 31, pp. 66-71.

Norihiro, K., Toshiharu, M. and Masaki, Y. (2010), "Printing fabrication of a Bucky Gel actuator/sensor and its application to three-dimensional patterned devices", Advanced Robotics, Vol. 24, pp. 1471-1487.

Otsuka, K. and Wayman, C.M. (Eds) (1998), Shape Memory Materials, Cambridge University Press, Cambridge, MA.

Richards, D., Tan, Y. and Jia, J., (2013), "3D printing for tissue engineering", Israel fournal of Chemistry, Vol. 53, pp. 805-814.

Rossiter, J. and Peter, W. (2009), "Printing 3D dielectric elastomer actuators for soft robotics", International Conference for Optics and Photonics, pp. 968-973.

Samuel, M., Michael, T. and Cagdas, D. (2013), "Robot self-assembly by folding: a printed inchworm robot", 2013 IEEE International Conference on Robotics and Automation, Karlsruhe, pp. 277-282.

Sun, L., Huang, W. and Ding, Z., (2012), "Stimulusresponsive shape memory materials: a review", Materials \& Design, Vol. 33, pp. 577-640.

Tibbits, S. (2014), " $4 \mathrm{D}$ printing: multi-material shape change", Architectural Design, Vol. 84, pp. 116-121.

Tibbits, S. and Cheung, K. (2012), "Programmable materials for architectural assembly and automation", Assembly Automation, Vol. 32, pp. 216-225. 
Tolley, M., Felton, S. and Miyashita, S. (2013), "Self-folding shape memory laminates for automated fabrication", International Conference on Robots and Systems, IEEE, St. Louis, MO, pp. 1049-1053.

Walton, Z. (2013), " $4 \mathrm{D}$ printing is the future of $3 \mathrm{D}$ printing and it's already here", WebPro News.

Wilhelm, S. and Curbach, M. (2014), "Review of possible mineral materials and production techniques for a building material on the moon", Structural Concrete, Vol. 15, pp. 419-428.

Won, S., Kyung, T. and Sung, H., (2016), "Review of biomimetic underwater robots using smart actuators", International fournal of Precision Engineering and Manufacturing, Vol. 13, pp. 1281-1292.

Wu, L., Jin, C. and Sun, X. (2010), "Synthesis, properties, and light-induced shape memory effect of multiblock polyesterurethanes containing biodegradable segments and pendant cinnamamide groups", Biomacromolecules, Vol. 12, pp. 235-241.

Xie, T. (2011), "Recent advances in polymer shape memory", Polymer, Vol. 52, pp. 4985-5000.

Yamagishi, Y., Masuda, T. and Takei, N. (2013), "Three-dimensional assembly of multilayered tissues, in first cirp conference on biomanufacturing", Rapid Prototyping Fournal, Vol. 5, pp. 201-204.

Ying, L., Boyles, J.K., Genzer, J. and Dickey, M.D. (2012), "Self-folding of polymer sheets using local light absorption", Soft Material, Vol. 8, pp. 1764-1769.

Zhang, S. (2011), "Molecular self-assembly", The Encyclopedia of Materials: Science and Technology, Vol. 9, pp. 5822-5829.

Zhang, S. (2013), "Building from bottom-up", Material Today, Vol. 6, pp. 20-27.
Zhang, S., Marini, D. and Hwang, W. (2012), "Designing nanobiological materials through self-assembly of peptide and proteins", Current Opinion in Chemical Biology, Vol. 6, pp. 865-871.

Zhu, Y., Hu, J. and Luo, H. (2012), "Rapidly switchable water-sensitive shape-memory cellulose/ elastomer nanocomposites", Soft Material, Vol. 8, pp. 2509-2517.

\section{Further reading}

Browne, A., Johnson, N. and Namuduri, C. (2004), "Electrostatically releasable fastening system and method of use”, US Patent Specification No. 6944920.

Li, D., He, J. and Tian, X., (2013), "Additive manufacturing: integrated fabrication of macro/microstructures", fournal of Mechanical Engineering, Vol. 49, pp. 129-135.

Tibbits, S. (2013), "4D Printing: multi-material shape change", Architectural Design: High Definition, pp. 116-121.

Wei, F., Zhang, H. and Zhang, X. (2006), "The application and development of intelligent materials", Materials Review, Vol. 20, pp. 375-379.

Xi, L., Zheng, J. and Zhang, H. (2003), "Intelligent materials: research state and prospects", Materials Review, Vol. 17, pp. 235-238.

Zhao, Y., Huang, W. and Wang, C. (2012), "Thermo/ chemo-responsive shape memory effect for micro/nano surface patterning atop polymers", Nanoscience and Nanotechnology Letters, Vol. 4, pp. 862-878.

\section{Corresponding author}

Jianzhong Shang can be contacted at: jz_shang_ nudt@yeah.net 